JOURNAL of

MAINE MEDICAL CENTER Journal of Maine Medical Center

\title{
Factors that Influence Perception of HIV Risk and Willingness to Use Pre-Exposure Prophylaxis in People Who Inject Drugs
}

\author{
Daria Egorova \\ Tufts University School of Medicine
}

Et al.

Follow this and additional works at: https://knowledgeconnection.mainehealth.org/jmmc

Part of the Virus Diseases Commons

\section{Recommended Citation}

Egorova, Daria; Fenton, Anny; and Thakarar, Kinna (2021) "Factors that Influence Perception of HIV Risk and Willingness to Use Pre-Exposure Prophylaxis in People Who Inject Drugs," Journal of Maine Medical Center. Vol. 3 : Iss. 2 , Article 5.

Available at: https://knowledgeconnection.mainehealth.org/jmmc/vol3/iss2/5 https://doi.org/10.46804/ 2641-2225.1084

The views and thoughts expressed in this manuscript belong solely to the author[s] and do not reflect the opinions of the Journal of Maine Medical Center or MaineHealth.

This Original Research is brought to you for free and open access by Maine Medical Center Department of Medical Education. It has been accepted for inclusion in the Journal of Maine Medical Center by an authorized editor of the MaineHealth Knowledge Connection. For more information, please contact Dina McKelvy mckeld1@mmc.org.

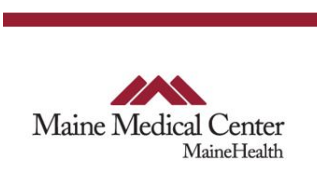




\section{Factors that Influence Perception of HIV Risk and Willingness to Use Pre- Exposure Prophylaxis in People Who Inject Drugs}

\section{Acknowledgements}

The authors would like to thank the study participants, as well as Kim Murray, MPP for their contributions to this study.

\section{Authors}

Daria Egorova, Anny Fenton, and Kinna Thakarar 


\title{
Factors that Influence Perception of HIV Risk and Willingness to Use Pre-Exposure Prophylaxis in People Who Inject Drugs
}

\author{
Daria Egorova, ${ }^{1}$ Anny Fenton, $\mathrm{PhD},{ }^{2}$ Kinna Thakarar, DO, $\mathrm{MPH}^{3}$ \\ ${ }^{1}$ Tufts University School of Medicine, Boston MA, ${ }^{2}$ Dana Farber/Harvard Cancer Center, Boston MA, ${ }^{3}$ Maine Medical Center, \\ Portland, ME
}
Introduction: Sexual and injection behaviors increase the risk of HIV transmission in people who inject drugs (PWID). We aimed to determine the prevalence of sexual and drug behaviors that increase HIV risk in PWID hospitalized for infections related to injection drug use in Maine. We also examined factors that influenced their perception of HIV risk and willingness to take pre-exposure prophylaxis (PrEP).

Methods: $\quad$ We surveyed 101 PWID with infections related to injection drug use who were hospitalized at 4 hospitals
in Maine. T-tests assessed differences in means of bacterial infection risk scores and willingness to take
PrEP based on different sociodemographic factors.

Results: $\quad$ PWID engaging in unsafe sexual behavior had a higher mean score of bacterial infection risk than those engaging in safer sexual behavior (3.90 vs $3.07 ; \mathrm{P}=.06)$. PWID with lower educational attainment had a lower mean score of willingness to take PrEP than those with a higher educational attainment (3.19 vs $3.85 ; P=.02$ ). Willingness to take PrEP was positively associated with the level of educational attainment (odds ratio, 2.14; $95 \% \mathrm{Cl}, 1.01-4.93 ; \mathrm{P}=.048$ ).
Discussion: $\quad$ Our findings associated willingness to take PrEP with educational attainment. To prevent HIV infection, harm reduction programs that discuss risk behaviors with PWID could be expanded, especially in rural areas where people have lower educational levels.

Conclusions: We found that injection and sexual risk behaviors co-occurred in PWID, PWID had an overall perception of low HIV risk, and willingness to take PrEP was positively associated with the level of educational attainment.

Keywords: $\quad$ HIV, substance use disorder, sexual behavior, pre-exposure prophylaxis, rural health services

$\mathrm{P}$ eople who inject drugs (PWID) are at risk for HIV infection and account for approximately $10 \%$ of new HIV diagnoses in the United States. ${ }^{1}$ Risk factors for HIV transmission among PWID include sharing drug-injection equipment, such as needles, syringes, and cookers. ${ }^{1}$ HIV transmission associated with unsafe injection practices can be minimized via access to syringe service programs (SSPs), in which PWID can exchange used needles and syringes for sterile ones. However, SSPs vary in distribution throughout the United States and are largely inaccessible to

Correspondence: Kinna Thakarar, DO, MPH

Maine Medical Center/TUSM

41 Donald Bean Drive, Suite B

South Portland, ME 04106

kthakarar@mmc.org
PWID from more rural areas like Maine. ${ }^{2,3}$ Several studies have noted that sexual risk behaviors (eg, having sex in exchange for money, inconsistent condom use, having multiple sexual partners) tend to co-occur with unsafe injection behaviors in PWID and may contribute to the spread of HIV. ${ }^{4}$

Prior studies also note that a constellation of psychosocial vulnerabilities (eg, stigma associated with drug use and HIV) may underlie why people either abstain from sex or pursue unsafe sex (with a relative unlikelihood to engage in safer sex). ${ }^{5}$ To reduce HIV incidence in PWID who are at high risk of HIV infection, the Centers for Disease Control and Prevention recommend a daily dose of oral preexposure prophylaxis (PrEP). ${ }^{6}$ However, people can face several challenges in accessing PrEP. Some 
reasons for low uptake and adherence include stigma associated with PrEP use, a perception of low HIV risk, and a daily dosing regimen of the drug. ${ }^{7}$ Providers also may not view injection drug use as a significant risk factor for HIV and consequently abstain from discussing PrEP and prescribing the drug to PWID.8,9

These challenges underscore the importance of optimizing PrEP uptake in PWID from largely rural states such as Maine, where there were only 7 operational SSPs in 2019-2020 when this study was conducted. In this study, we aimed to better understand risk-taking behaviors and attitudes toward PrEP use among PWID. We set out to determine the prevalence of sexual and drug behaviors that increase HIV risk for PWID and examine factors that influence the perception of HIV risk and willingness to take PrEP among PWID in a rural state.

\section{METHODS}

\section{Study design}

This study is a cross-sectional analysis of PWID with infections associated with injection drug use who were hospitalized at 4 hospitals in Maine between January, 2019 and March, 2020.3 Data were collected through the electronic health record and an audio computer-assisted survey as part of a larger initial 18-month cross-sectional study.3 We focused our data analysis on survey questions specific to sexual behaviors, injection practices, HIV awareness, and attitudes toward drug programs. The MaineHealth Institutional Review Board approved this study.

\section{PRIMARY OUTCOME}

The primary outcome was willingness to use PrEP, which was measured by answers to the question, "How willing would you be to take a pill a day (preexposure prophylaxis, ie, PrEP) if you thought it would decrease your chances of getting HIV?" Responses were on a 5-point Likert scale ranging from "very unwilling" to "very willing" (Table 1).

\section{VARIABLES}

Demographics. "Rural" was defined as "isolated rural," "small rural," and "large rural." An area's rurality was determined based on the ruralurban commuting area (RUCA) code associated with a participant's ZIP code. ${ }^{10}$ Insurance was collected through the electronic health record and categorized. A collapsed category of "Insured" participants was created. This category consisted of anyone who stated that they are insured under "Medicaid," "Medicare," "Dual Medicare/Medicaid," or through "Commercial" insurance. "Uninsured" participants were those who reported they had "No insurance."

Secondary outcome. The Bacterial Infections Risk Scale for Injectors (BIRSI)-7, a 7-item index, was used to measure unsafe injection behavior, such that a higher score indicated poorer hygiene and practice. ${ }^{11}$ The BIRSI-7 score was based on a combination of different injection practices, such as the frequency of re-using cookers and filters, handwashing before injecting, and cleaning skin before injecting.

Independent variables. Unsafe sexual behavior was assessed based on yes/no responses to behavioral questions about using condoms during the last sexual encounter; not having sex in exchange for money, drugs, or a place to stay; and not having sex with other PWID. Respondents were classified as engaging in "unsafe sexual behavior" if they answered yes to engaging in at least one unsafe sexual behavior. Perception of HIV risk was categorized as "low" if a participant answered that they are "very unlikely" or "somewhat unlikely" to become infected with HIV from either injecting drugs or having sex in the next 6 months. Perception of HIV risk was categorized as "high" if participants answered that they are "very likely," "somewhat likely," or "neither likely nor unlikely" to become infected with HIV. Other variables in Table 1 were collected via self-report.

\section{STATISTICAL ANALYSIS}

T-tests were conducted to compare differences between means of PrEP willingness and sociodemographic characteristics, such as sex, sexual orientation, experiencing homelessness, health insurance status, RUCA codes, educational attainment, and perception of HIV risk from injection behavior and sexual behavior. Separate t-tests were also performed to establish differences in means of BIRSI-7 scores between PWID with safer sexual behavior versus those with unsafe sexual behavior. 
Table 1. Select Characteristics of Study Participants

\begin{tabular}{|c|c|}
\hline Sociodemographic & Data value* \\
\hline \multicolumn{2}{|l|}{ Sex, N (\%) } \\
\hline Male & $44(44)$ \\
\hline Female & $57(56)$ \\
\hline Age, y, median (IQR) & $35(31-40)$ \\
\hline \multicolumn{2}{|l|}{ Race, N (\%) } \\
\hline White & $95(94)$ \\
\hline Black & $1(1)$ \\
\hline Hispanic & $0(0)$ \\
\hline Unknown & $2(2)$ \\
\hline Other & $3(3)$ \\
\hline \multicolumn{2}{|l|}{ Sexual orientation, $N(\%)$} \\
\hline Heterosexual & $83(82)$ \\
\hline Bisexual & $16(16)$ \\
\hline Gay or lesbian & $2(2)$ \\
\hline Experiencing homelessness, $\mathrm{N}(\%)$ & $46(46)$ \\
\hline \multicolumn{2}{|l|}{ RUCA codes, $N(\%)$} \\
\hline Urban & $66(65)$ \\
\hline Rural & $35(35)$ \\
\hline \multicolumn{2}{|l|}{ Educational attainment, $\mathrm{N}(\%)$} \\
\hline High school, GED, or less & $62(61)$ \\
\hline 2- to 4-year college degree or some college (no degree) & $39(39)$ \\
\hline \multicolumn{2}{|l|}{ Health insurance status ${ }^{\dagger}, \mathrm{N}(\%)$} \\
\hline Uninsured & $25(25)$ \\
\hline Insured & $74(75)$ \\
\hline \multicolumn{2}{|l|}{ Injection risk behavior } \\
\hline BIRSI-7 score, average (SD) & $3.77(1.61)$ \\
\hline \multicolumn{2}{|l|}{ Sexual risk behavior } \\
\hline Number of sexual contacts 30 days before hospitalization, median & 1 \\
\hline No condom during last sexual encounter, N (\%) & $78(78)$ \\
\hline Sex in exchange for money/drugs/place to stay, N (\%) & $12(12)$ \\
\hline Sex with people who inject drugs, N (\%) & $45(46)$ \\
\hline \multicolumn{2}{|l|}{ HIV testing } \\
\hline Ever tested for HIV, N (\%) & $95(95)$ \\
\hline How many times tested, average & 4 \\
\hline \multicolumn{2}{|l|}{ Willingness to take PrEP, N (\%) } \\
\hline Very willing & $31(31)$ \\
\hline Somewhat willing & $23(23)$ \\
\hline Neutral & $23(23)$ \\
\hline Somewhat unwilling & $8(8)$ \\
\hline Very unwilling & $16(16)$ \\
\hline Discussed PrEP with health care provider & $8(8 \%)$ \\
\hline
\end{tabular}


Table 2. PrEP Willingness by Patient Characteristics

\begin{tabular}{|c|c|c|}
\hline Patient characteristics & $\begin{array}{l}\text { Willingness to } \\
\text { take PrEP* }\end{array}$ & $P$ value \\
\hline \multicolumn{2}{|l|}{ Sex } & \multirow{3}{*}{.43} \\
\hline Female $(\mathrm{N}=57)$ & 3.54 & \\
\hline Male $(\mathrm{N}=44)$ & 3.32 & \\
\hline \multicolumn{2}{|l|}{ Sexual Orientation } & \multirow{3}{*}{.47} \\
\hline Heterosexual $(N=83)$ & 3.40 & \\
\hline Bisexual, gay, or lesbian $(\mathrm{N}=18)$ & 3.67 & \\
\hline \multicolumn{2}{|l|}{ Experiencing homelessness } & \multirow{3}{*}{.83} \\
\hline Yes $(N=46)$ & 3.48 & \\
\hline No $(N=55)$ & 3.42 & \\
\hline \multicolumn{2}{|l|}{ Health insurance status } & \multirow{3}{*}{.83} \\
\hline Insured $(\mathrm{N}=74)$ & 3.43 & \\
\hline Uninsured $(\mathrm{N}=25)$ & 3.36 & \\
\hline \multicolumn{2}{|l|}{ RUCA codes } & \multirow{3}{*}{.03} \\
\hline Rural $(\mathrm{N}=35)$ & 3.03 & \\
\hline Urban $(\mathrm{N}=66)$ & 3.67 & \\
\hline \multicolumn{2}{|l|}{ Education } & \multirow{3}{*}{.02} \\
\hline High school, GED, or less $(\mathrm{N}=62)$ & 3.19 & \\
\hline Some college or 2 - to 4 -year degree $(\mathrm{N}=39)$ & 3.85 & \\
\hline \multicolumn{2}{|l|}{ Perception of HIV risk from injection behavior } & \multirow{3}{*}{.23} \\
\hline Low $(\mathrm{N}=84)$ & 3.41 & \\
\hline $\operatorname{High}(\mathrm{N}=8)$ & 4.00 & \\
\hline \multicolumn{2}{|l|}{ Perception of HIV risk from sexual behavior } & \multirow{3}{*}{.68} \\
\hline Low $(\mathrm{N}=88)$ & 3.45 & \\
\hline $\operatorname{High}(\mathrm{N}=4)$ & 3.75 & \\
\hline
\end{tabular}

Abbreviations: GED, General Educational Development; PrEP, pre-exposure prophylaxis; RUCA, rural-urban commuting area.

*Willingness to take PrEP was based on responses to a 5-point Likert scale ranging from "very unwilling" to "very willing." A higher number corresponds to a higher willingness to take PrEP once daily to decrease the chances of HIV infection. 
To test whether certain sociodemographic factors contributed more than others to observed differences, an ordered logit was performed. The primary outcome was willingness to use PrEP, controlling for rurality and educational attainment. These 2 sociodemographic factors were chosen for the ordered logit based on previous studies examining sexual risk among PWID.,8 For other covariates that were considered in the original model, refer to Table 1. All analyses were performed using Stata Version 16.0. ${ }^{12}$

\section{RESULTS}

This study included 101 participants. Among the participants, 31\% stated that they were "very willing" and $23 \%$ stated that they were "somewhat willing" to take PrEP. Also, $8 \%$ of participants stated that they were "somewhat unwilling" and $16 \%$ of participants stated that they were "very unwilling" to take PrEP (Table 1). PWID with lower educational attainment status (high school, GED [General Educational Development], or less) had a lower mean score of willingness to take PrEP than those with higher educational attainment (3.19 vs 3.85; $\mathrm{P}=.02)$. PWID from rural areas had a lower mean score of PrEP willingness than those from urban areas (3.03 vs $3.67 ; \mathrm{P}=.03$ ). Among the participants, $84 / 92$ $(91 \%)$ had a perception of low HIV risk from injecting drugs, and $88 / 92(96 \%)$ had a perception of low
HIV risk from sexual behavior (Table 2). Individuals engaging in unsafe sexual behavior $(\mathrm{N}=83)$ had a marginally higher mean BIRSI-7 score than those engaging in safer sexual behavior $(\mathrm{N}=15)$ (3.07 vs 3.90; $P=.06$ ) (Figure 1). In our regression model, willingness to take PrEP was positively associated with the level of educational attainment (adjusted odds ratio, $2.14 ; 95 \% \mathrm{Cl}, 1.01-4.93, \mathrm{P}=.048)$.

\section{DISCUSSION}

Findings from this study with hospitalized PWID suggest that PWID with a lower level of educational attainment are less willing to take PrEP to decrease their chances of HIV infection. Hospitalized PWID from rural areas are similarly less willing to take PrEP. Our regression model indicated that willingness to take PrEP was positively associated with level of educational attainment. The perception of HIV risk from injection drug use and sexual behavior was low among hospitalized PWID, although most PWID reported engaging in unsafe sexual behavior and unsafe injection drug use. PWID engaging in safer sexual behavior had a lower BIRSI than those engaging in unsafe sexual behavior, although this finding was not significant.

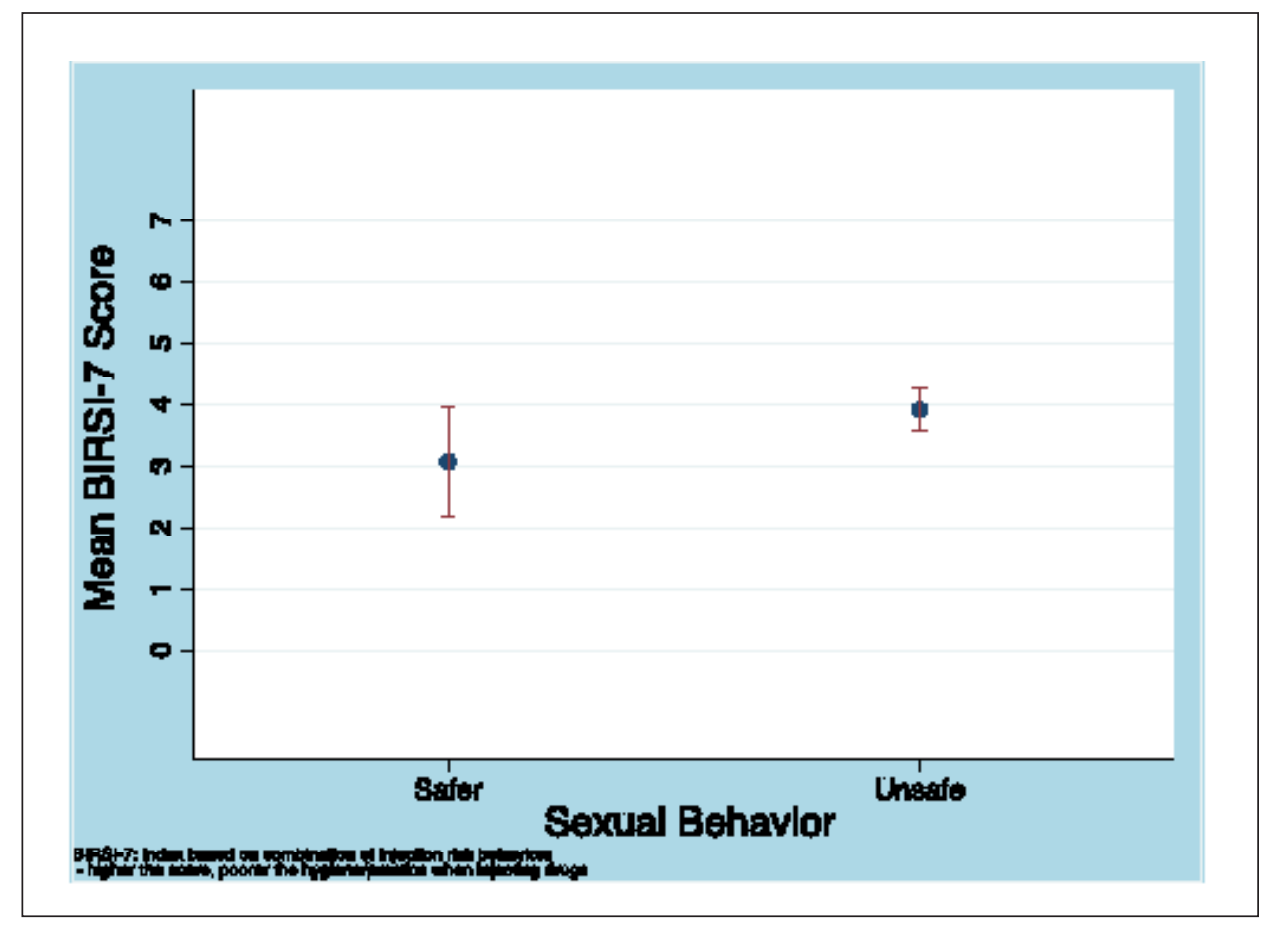

Figure 1. Co-occurrence of Injection and Sexual-Related Risk Behavior in PWID ( $N=101)$. Abbreviations: BIRSI-7, Bacterial Infections Risk Scale for Injectors 


\section{IMPLICATIONS}

The positive association between educational attainment and willingness to take PrEP highlights the importance of targeting future HIV prevention efforts toward PWID in rural areas, where individuals have disproportionately lower educational levels. The perception of low HIV risk among PWID who participate in unsafe sexual and injection-related behaviors and across different sociodemographic characteristics supports the importance of expanding SSPs throughout Maine. These programs discuss risk behavior with PWID and could help this population understand why they are at high risk for HIV.3 Given the co-occurrence of injection and sexual-related risk behaviors in PWID, our findings support that comprehensive efforts to prevent HIV in PWID should include evaluating sexual risk for HIV transmission.

\section{Limitations}

Our study had several limitations. Our sample size was small $(\mathrm{N}=101)$ and underpowered to address our study question. Not all participants answered every survey question, decreasing the sample size for our analyses. Notably, only 92 participants responded to the questions regarding HIV risk perception from drug use/sexual behavior. Also, only 98 participants responded to questions regarding condom use during their last sexual encounter; having sex in exchange for money, drugs, or a place to stay; and having sex with people who inject drugs. We attribute the lack of statistical significance in mean BIRSI-7 scores between PWID engaging in safer sexual behavior versus those engaging in unsafe sexual behavior to the small sample size.

Although the PWID in this our may be good representatives of groups at risk for HIV in Maine, findings from our population may not be generalizable to other geographic regions within the United States. Similarly, because our participants were all hospitalized with an injectionrelated infection, our sample may not represent PWID who are at lower risk of developing infectious complications from drug use. Most participants in this study self-identified as white and heterosexual, further limiting generalizability to more diverse populations. Further research would benefit from sampling a larger, more diverse population of PWID to assess the prevalence of HIV-related risk behaviors and interest in PrEP.

\section{CONCLUSIONS}

Our findings suggest overlap in the co-occurrence of injection-related and sexual-related risk behavior in PWID. We also noted an overall perception of low HIV risk in PWID, despite participation in unsafe behavior, and we provided information about factors that may influence willingness to use PrEP.

\section{ACKNOWLEDGMENTS}

We thank the study participants, as well as Kim Murray, MPP, for their contributions to this study.

\section{Conflict of interest: none}

Financial Disclosure: The research reported was supported by grant U54 GM115516 from the National Institutes of Health for the Northern New England Clinical and Translational Research network.

\section{REFERENCES}

1. Centers for Disease Control and Prevention. HIV Surveillance Report, 2018 (Updated). Vol 31. Published May 2020. Accessed June 18，2020. http://www.cdc.gov/hiv/library/reports/hivsurveillance.html

2. Aspinall EJ, Nambiar D, Goldberg DJ, et al. Are needle and syringe programmes associated with a reduction in HIV transmission among people who inject drugs: a systematic review and metaanalysis. Int J Epidemiol. 2014;43(1):235-248. doi:10.1093/ije/ dyt243

3. Thakarar K, Murray K, Carwile J, et al. Injections and infections: understanding harm reduction utilization in a rural state. Open Forum Infect Dis. 2020;7(Suppl 1):S715-S716. doi:10.1093/ofid/ ofaa439.1600

4. Bogart LM, Kral AH, Scott A, et al. Sexual risk among injection drug users recruited from syringe exchange programs in California. Sex Transm Dis. 2005;32(1):27-34. doi:10.1097/01. olq.0000148294.83012.d0

5. Miller CT, Solomon SE, Bunn JY, Varni SE, Hodge JJ. Psychological symptoms are associated with both abstinence and risky sex among men with HIV. Arch Sex Behav. 2015;44(2):453-465. doi:10.1007/s10508-014-0464-2

6. Centers for Disease Control and Prevention (CDC). Update to Interim Guidance for Preexposure Prophylaxis (PrEP) for the Prevention of HIV Infection: PrEP for injecting drug users. MMWR Morb Mortal Wkly Rep. 2013;62(23);463465. Accessed January 30, 2021. https://www.cdc.gov/mmwr/ preview $/ \mathrm{mmwrhtml} / \mathrm{mm} 6223 \mathrm{a} 2 . \mathrm{htm}$

7. Sidebottom D, Ekström AM, Strömdahl S. A systematic review of adherence to oral pre-exposure prophylaxis for HIV - how can we improve uptake and adherence? BMC Infect Dis. 2018;18(1):581. doi:10.1186/s12879-018-3463-4

8. Edeza A, Bazzi A, Salhaney P. HIV pre-exposure prophylaxis for people who inject drugs: the context of co-occurring injectionand sexual- related HIV risk in the U.S. Northeast. Subst Use Misuse. 2020;55(4):525-533. doi:10.1080/10826084.2019.1673 419 


\section{Egorova et al.: Perception of HIV Risk and Willingness to Use PrEP in PWID}

9. Krakower DS, Beekmann SE, Polgreen PM, Mayer KH. Diffusion of newer HIV prevention innovations: variable practices of frontline infectious diseases physicians. Clin Infect Dis. 2016;62(1):99-105. doi:10.1093/cid/civ736

10. Economic Research Service. Rural-urban commuting area codes. U.S. Department of Agriculture. Updated August 17, 2020. Accessed January 30, 2021, https://www.ers.usda.gov/ data-products/rural-urban-commuting-area-codes
11. Phillips KT, Anderson BJ, Herman DS, Liebschutz JM, Stein MD. Risk factors associated with skin and soft tissue infections among hospitalized people who inject drugs. J Addict Med. 2017;11(6):461-467. doi:10.1097/ADM.0000000000000346

12. Stata 16. Version 16. StataCorp. 2019. Accessed January 30, 2021. http://www.stata.com/ 